\title{
Characteristics of the CsI:Tl Scintillator Crystal for X-Ray Imaging Applications
}

\author{
Maria da Conceição Costa Pereira, Tufic Madi Filho, José Roberto Berretta, \\ Carlos Henrique de Mesquita
}

Nuclear and Energy Research Institute-IPEN/CNEN-SP, São Paulo, Brazil

Email: macoper@ipen.br

How to cite this paper: Pereira, M. da C.C., Filho, T.M., Berretta, J.R. and de Mesquita, C.H. (2018) Characteristics of the CsI:Tl Scintillator Crystal for X-Ray Imaging Applications. Materials Sciences and Applications, 9, 268-280.

https://doi.org/10.4236/msa.2018.92018

Received: October 17, 2017

Accepted: February 4, 2018

Published: February 7, 2018

Copyright (c) 2018 by authors and Scientific Research Publishing Inc. This work is licensed under the Creative Commons Attribution International License (CC BY 4.0).

http://creativecommons.org/licenses/by/4.0/

\begin{abstract}
Scintillators are high-density luminescent materials that convert X-rays to visible light. Thallium doped cesium iodide (CsI:Tl) scintillation materials are widely used as converters for X-rays into visible light, with very high conversion efficiency of 64.000 optical photons/MeV. CsI:Tl crystals are commercially available, but, the possibility of developing these crystals into different geometric shapes, meeting the need for coupling the photosensor and reducing cost, makes this material very attractive for scientific research. The objective of this work was to study the feasibility of using radiation sensors, scintillators type, developed for use in imaging systems for X-rays. In this paper, the CsI:Tl scintillator crystal with nominal concentration of the $10^{-3} \mathrm{M}$ was grown by the vertical Bridgman technique. The imaging performance of CsI:Tl scintillator was studied as a function of the design type and thickness, since it interferes with the light scattering and, hence, the detection efficiency plus final image resolution. The result of the diffraction X-ray analysis in the grown crystals was consistent with the pattern of a face-centered cubic (fcc) crystal structure. Slices $25 \times 2 \times 3 \mathrm{~mm}^{3}$ (length, thickness, height) of the crystal and mini crystals of $1 \times 2 \times 3 \mathrm{~mm}^{3}$ (length, thickness, height) were used for comparison in the imaging systems for X-rays. With these crystals scintillators, images of undesirable elements, such as metals in food packaging, were obtained. One-dimensional array of photodiodes and the photosensor CCD (Coupled Charge Device) component were used. In order to determine the ideal thickness of the slices of the scintillator crystal CsI:Tl, Monte Carlo method was used.
\end{abstract}

\section{Keywords}

Crystal Growth, Scintillator, X-Ray, Imaging Systems, Photodiodes, Coupled Charge Device 


\section{Introduction}

X-rays were first discovered by Wilhelm Roentgen in Germany, in 1895 [1]. The constant and increasing number of applications of X-rays in many fields of human activity has stimulated the research in scintillation materials. There has been a rise in this field, over the last 10 years, induced by new demanding applications of medical, industrial and scientific imaging together with the development and exploitation of powerful X-ray sources, such as synchrotron radiation. Thus, intense research and development [2] [3] have continued, looking for new scintillation materials or the optimization of the current ones, taking advantage of new technological methods for their preparation. Understanding the underlying physical mechanisms of energy transfer and storage and the role of particular material defects is of crucial importance for bringing the materials performance close to their intrinsic limits. Further progress in the achievement of the ideal detector design is related to the rapid development in the field of photodetectors, which are an indispensable part of scintillation detectors [4].

Detectors for X-ray application cover a broad range including Si detectors, compound detectors and single crystal detectors. In the low energy X-ray region from a few hundred $\mathrm{eV}$ to about $20 \mathrm{KeV}$, direct detectors such as Si PIN photodiodes, Si APDs, and CCD (Charge Coupled Device) area image sensors are utilized [5] [6]. These detectors provide high detection efficiency and high energy resolution, and, hence, they are used in X-ray analysis, X-ray astronomical observation and physics experiments. The hard X-ray region with energy higher than soft X-rays is utilized in industrial and medical equipment due to the high penetration efficiency through objects. Scintillator detectors are widely used in these applications. These detectors use scintillators to convert X-rays into light and detect this light to identify X-rays indirectly. Especially in the medical field, the digital X-ray method, which uses X-ray detectors with large photosensitive area, is becoming mainstream, replacing the conventional film based method.

Indirect digital X-ray imaging requires scintillator material and some imaging sensor, such as photodiodes, CCD and CMOS imaging devices, for diagnostic and industrial applications. Thus, among the CsI:Tl inorganic scintillators crystal was elected in our research on the basis of the common favorable characteristics as a scintillator: (a) good mechanical strength, (b) it is slightly hygroscopic and (c) its emission spectrum light coincides with the visible region: it is, therefore, suitable to use of CCD and photodiodes system [7].

Our objective was to study the feasibility of using radiation sensors, type scintillators, developed in our laboratories, for use in imaging systems for X-rays. In this paper, the CsI:Tl scintillator crystal with nominal concentration of the $10^{-3}$ $\mathrm{M}$, grown by the vertical Bridgman technique was used in order to convert the $\mathrm{X}$ radiation into light photons. For this purpose, one-dimensional array of photodiodes and the photosensor CCD component, mentioned in the previous paragraph, was used. Monte Carlo method exclusive software was used [8] for to get scintillator crystal thickness and the energy absorption percentage of the gamma 
photon from a ${ }^{241} \mathrm{Am}$ source.

\section{Methods}

The crystal growth was accomplished by means of the vertical Bridgman technique [9] [10] [11] [12]. The starting material, cesium iodide (CsI) and thallium iodide (TII) were used with purity of $99.99 \%$. The concentration of the thallium doping element ( $\mathrm{Tl}$ ) was $10^{-3} \mathrm{M}$.

The crystals were cut in the desired dimensions for each type of experiment with a disk saw with diamond edge (Buehler ISOMET 11-1180). The cutting was done slowly, avoiding mechanical shock. The crystals were polished with ethylene glycol p.A. $\left(\mathrm{C}_{2} \mathrm{H}_{6} \mathrm{O}_{2}\right)$. The side surfaces were unpolished, to enhance internal reflection. The CsI:Tl crystals were cut and polished in the following dimensions: (a) Slices of $25 \times 2 \times 3 \mathrm{~mm}^{3}$ (length, thickness, height); (b) mini crystals of $1 \times 2 \times 3 \mathrm{~mm}^{3}$ (length, thickness, height); the mini crystals were introduced into the16 holes of the mini crystals support. Figure 1 shows the $25 \mathrm{~mm}$ support lenght.

The diffraction analysis of X-ray CsI:Tl crystals in, order to determine the crystal structure, was carried out by the powder diffraction method, at room temperature, using a diffractometer (Rigaku RINT2000 model) in following conditions: radiation: $\mathrm{k}$-alpha $\mathrm{Cu}$, scan rate: $2^{\circ} \mathrm{min}^{-1}$, voltage: $40 \mathrm{kV}$, current: $30 \mathrm{~mA}$.

In order to determine the ideal thickness of the slices of the scintillator crystal CsI:Tl, Monte Carlo method was used. The absorption of the X-ray energies in the scintillator was estimated by the Monte Carlo method. This method simulates all occurrences of gamma radiation interaction in the material media. The following interactions of the electromagnetic radiation taken into account were: Photoelectric effect, Compton scattering and Rayleigh scattering. Based on these interactions, a program was developed to simulate the interactions in the scintillator block and to estimate the level of absorption of the radiation $\mathrm{X}$, as a function of the dimensions of the scintillator. Figure 2 shows the main panel of the program.

The photodiodes dimensional arrangement units were mounted in a $200 \mathrm{~mm}$ line to cover the entire width of the mat of drag the sample to be analyzed. Another alternative used was the use of the CCD chip type, Sony model ILX551B 2048-pixel Linear Sensor (B/W) which gives the work more simple electronic circuitry.



Figure 1. Support mini-crystals. 


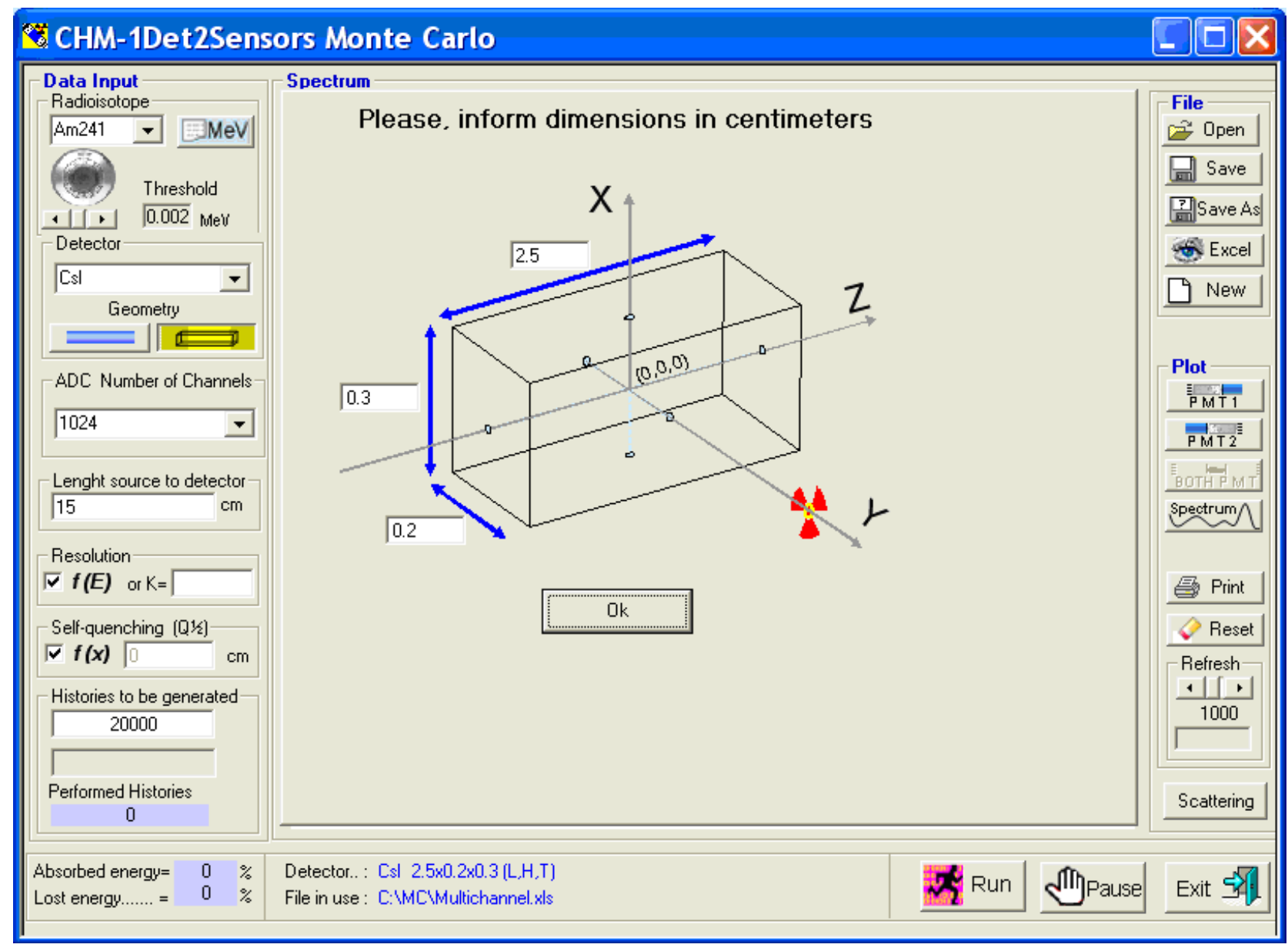

Figure 2. Main panel of Monte Carlo program used to estimate the optimized dimensions of the blocks (slices or mini crystals) of crystal scintillator.

Figure 3 shows the general scheme of the scanner used in this work. It is constituted by a tube $\mathrm{X}$-ray high-voltage source, able to feed it to $110 \mathrm{kV}$ to $0.3 \mathrm{~A}$. The tube and the high voltage generating unit are kept in the bottom of the equipment with shielding lead sheet and iron sheet, what does not allow X-ray leak to the environment. Above the X-ray tube, there is a collimator system that only allows flow of $\mathrm{X}$-rays to pass in an opening of $2 \mathrm{~mm}$, reaching only the area containing the scintillator. The unit has a mat where the objects to be analyzed move and pass through the X-ray beam. The entire system is controlled by a PC line computer, operating on the windows XP system. In this project, the detectors to generate pictures were constituted of a set of 6 units, with $16 \times 1$ photodiodes in one-dimensional arrangement, totaling 96 photodiodes linearly juxtaposed. One of the central units was reserved to introduce CsI:Tl scintillator. Figure 3(b).

\section{Results and Discussion}

Among the X- ray and gamma-ray converters in light photons, known as scintillators, the most efficient emits photons with wavelengths close to $400 \mathrm{~nm}$. 


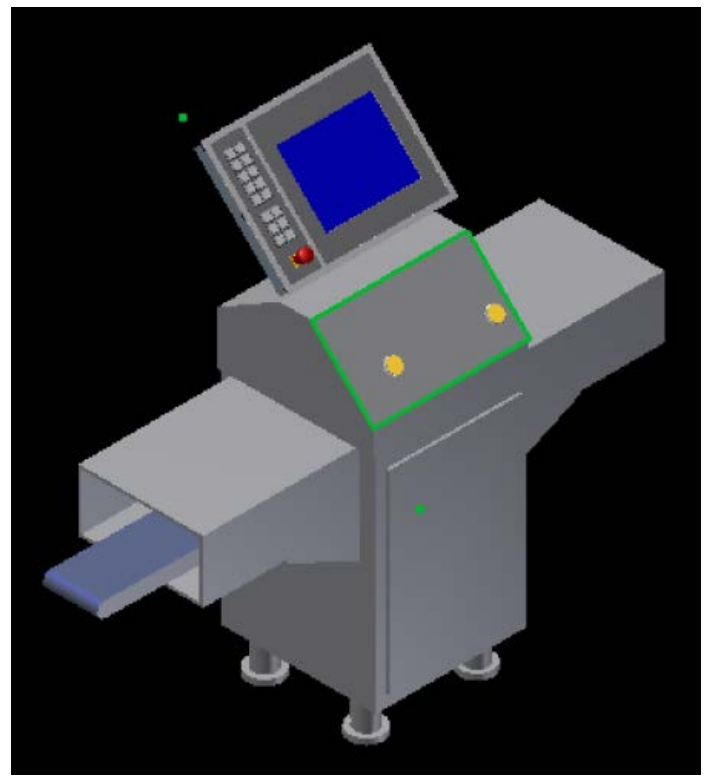

(a)

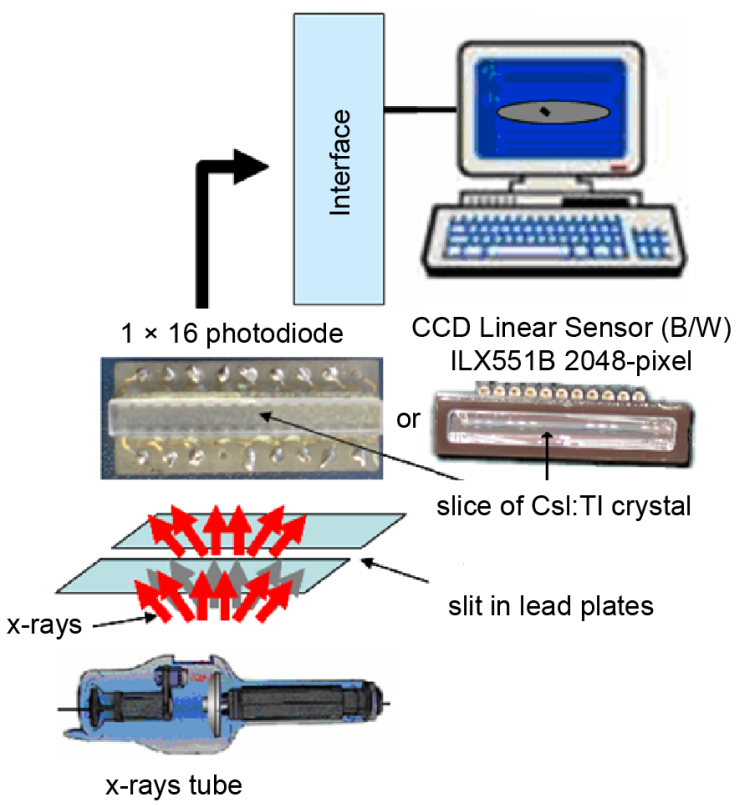

(b)

Figure 3. (a) Schematic representation of the scanner X-ray used in the experiments, showing the belt through which the objects to be analyzed; (b) Scheme of the scanner operating principle is shown.

Particularly among them, the CsI:Tl crystal has the best fit between the light emission spectrum (peak at $540 \mathrm{~nm}$ ) and the quantum sensitivity curve of the photodiodes. This explains the renewed interest in using this crystal as a scintillator.

Figure 4 shows CsI:Tl crystal with molar ratio $[\mathrm{Tl}] /[\mathrm{CsI}]=10^{-3}$ grown by the Bridgman technique, at a rate of $1 \mathrm{~mm} \cdot \mathrm{h}^{-1}$. The crystals were cut and polished in the following dimensions: slices $25 \mathrm{~mm}$ in length, $3 \mathrm{~mm}$ in width and $2 \mathrm{~mm}$ 
thickness; the mini crystal was cut and polished in the dimensions of $1 \times 2 \times 3$ $\mathrm{mm}^{3}$ (width, length, thickness).

$\mathrm{X}$-ray diffraction analysis demonstrated that the crystalline lattice of the crystal grown by the Bridgman technique resulted in a centered face cubic crystalline structure ( $\mathrm{cfc}$ ). This evidence can be established by comparing the X-ray diffraction pattern with that described by the tables (US Joint Committee on Powder Diffraction Standards) [13]. The complete diffraction pattern is shown in Figure 5 , which corresponds to the structure of CsI.

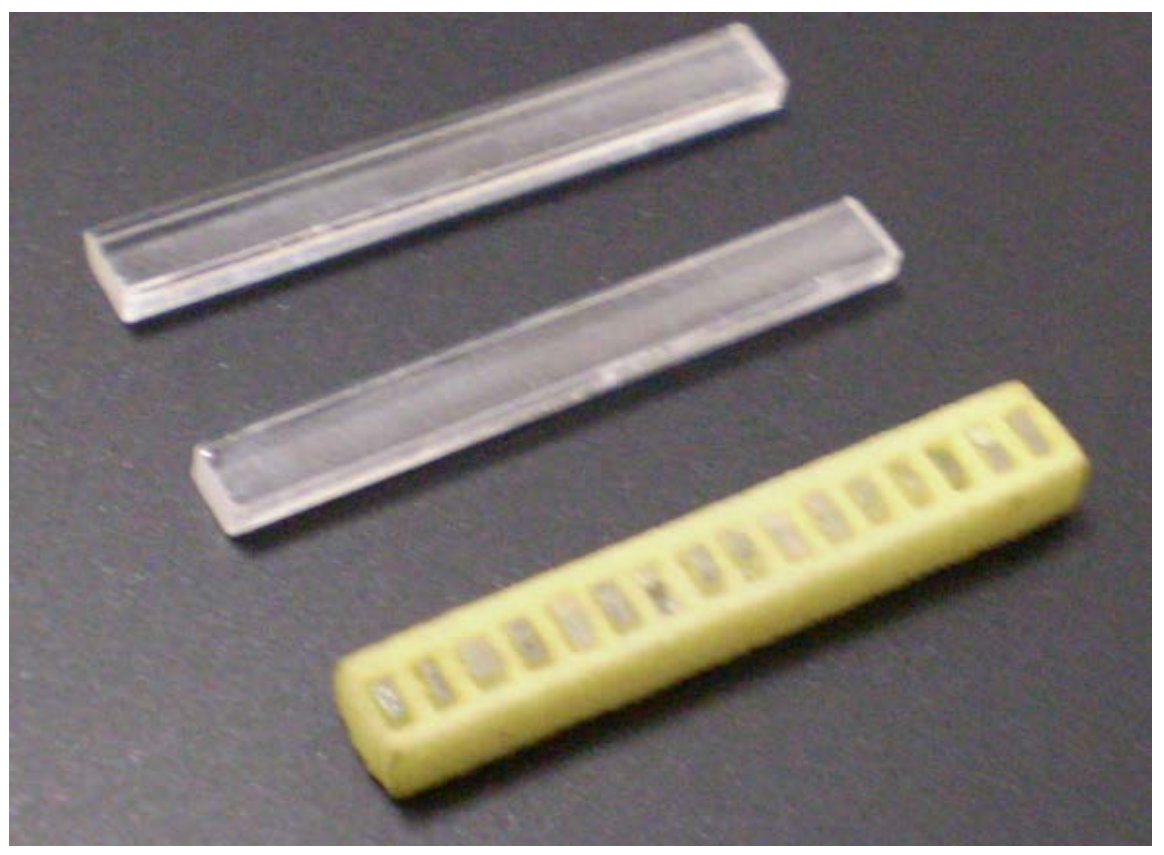

Figure 4. Slices of CsI:Tl crystal and support filled with mini crystals.

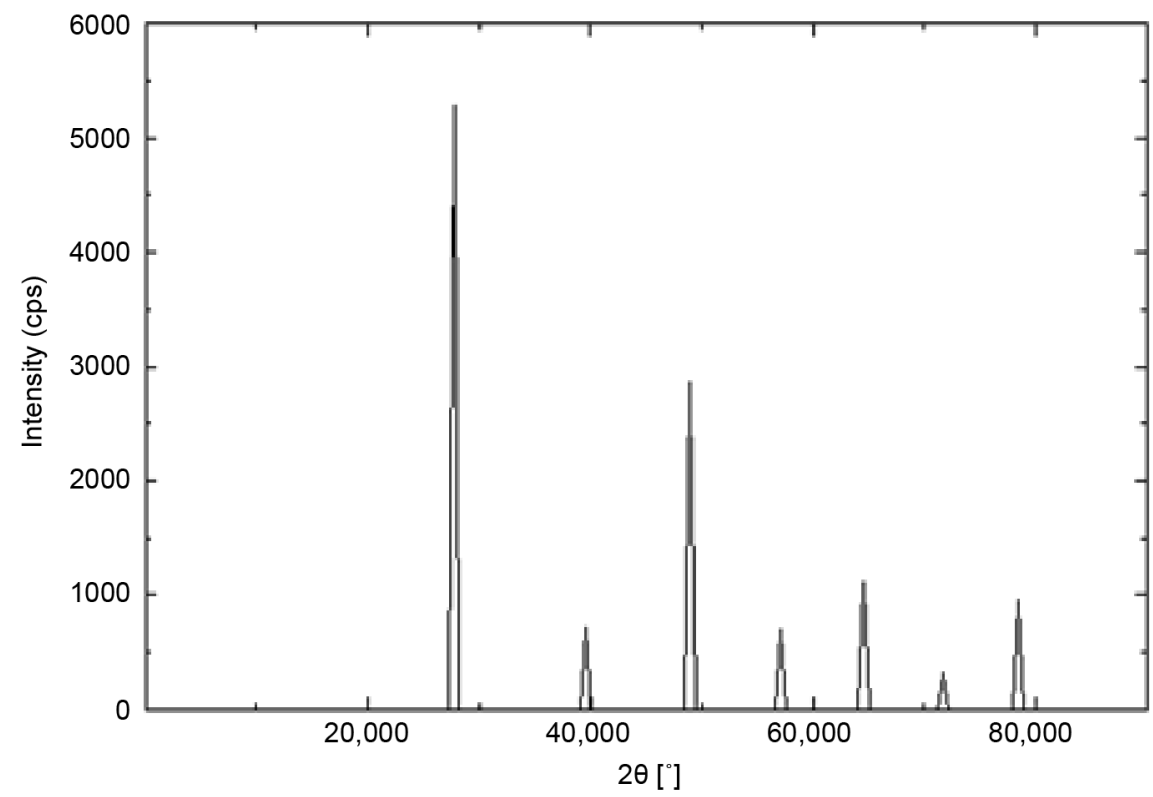

Figure 5. Complete X-ray diffraction pattern of the CsI:Tl crystal. 
The main purpose of the CsI:Tl crystal is its use in converting high energy photons from gamma rays and X-rays into light photons capable of sensitizing the photosensors. In order to meet this objective, the CsI:Tl crystal offers adequate conditions mainly because it consists of chemical elements with a high atomic masses $\left(Z_{\text {cesium }}=133, Z_{\text {iodine }}=127\right)$ and, therefore, favouring interactions of $X$ radiation with the multiple electron layers (photoelectric effect and Compton). The results of the simulation analysis using the Monte Carlo method are a convincing demonstration of the use of these crystals.

The results simulated by the Monte Carlo method relate the scintillator crystal thickness and the energy absorption percentage of the gamma photon energies of an Americium-241 source $\left({ }^{241} \mathrm{Am}\right)$. This source was used because it has the emission spectrum of energy (Table 1) close to the X-ray spectrum of the system used, especially, when operated with voltages from $30 \mathrm{kV}$ to $100 \mathrm{kV}$.

The Figure 6, show the main interaction effects of the radiation with the scintillator crystal of density $4.51 \mathrm{~g} / \mathrm{cm}^{3}$ as a function of the energy photon gamma emitted by a ${ }^{241} \mathrm{Am}$ source. As can be verified by the curves, the predominant effect is the photoelectric effect. The Compton effect contributed little to the interactions with the photons energy range $13.9 \mathrm{keV}$ (occurrence 42.7\%) and 59.5 $\mathrm{keV}$ (occurrence 35.9\%).

Table 2 shows the prediction of radiation absorption determined by the Monte Carlo method for CsI:Tl scintillation crystals, in the form of, mini crystals with $3 \times 1 \mathrm{~mm}^{2}$ (width, length, thickness $\mathrm{X}$ ) juxtaposed to a matrix arrangement and in the form of a $25 \times 3 \mathrm{~mm}^{2}$ slice, thickness $\mathrm{X}$, as shown in Table 2 .

Figure 7 and Figure 8 correspond to the graphical interpretation of the results shown in Table 2.

For the CsI:Tl crystals, at each thickness, the increase of $0.0874 \mathrm{~mm}$ doubles the absorption of the X-rays.

Two electronic boards were specially designed for the purpose of using the scintillation crystals, which convert light produced into electrical signals and, subsequently, through these plates the data is digitized and acquired by the PC computer.

Figure 9 shows part of the developed plate, with emphasis on the central part, where the scintillators were coupled. Two scintillation crystal couplings were

Table 1. Energy photon gamma emitted by an ${ }^{241} \mathrm{Am}$ source.

\begin{tabular}{cc}
\hline${ }^{241}$ Am Gamma photon Frequency of emission (Yield) (\%) & Energy (keV) \\
\hline 42.7 & 13.9 \\
2.4 & 26.3 \\
0.106 & 33.2 \\
35.9 & 59.5 \\
0.18 & 69.2 \\
\hline
\end{tabular}


M. da C. C. Pereira et al.



(a)

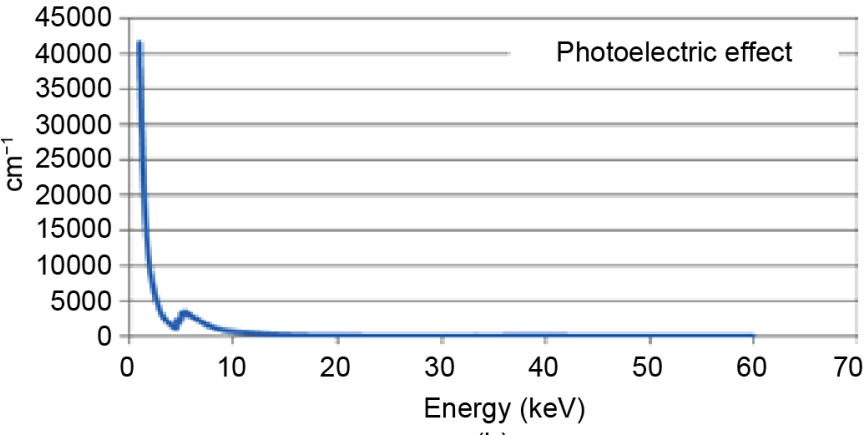

(b)

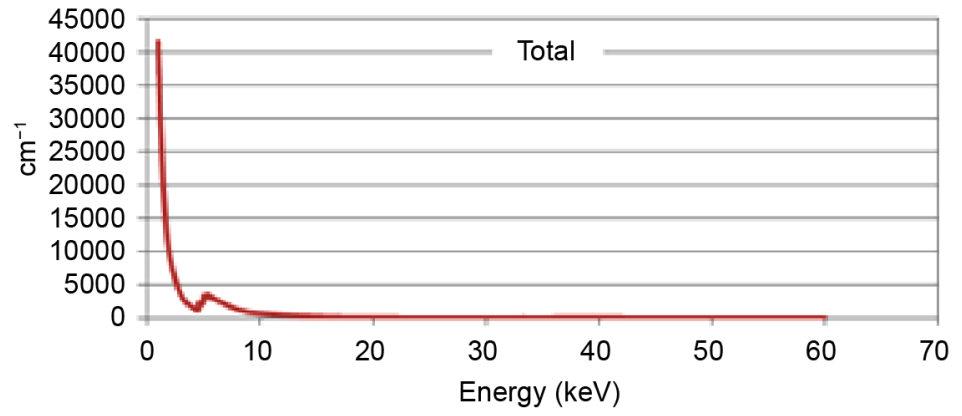

(c)

Figure 6. The main interaction effects of the radiation with the scintillator crystal.

Table 2. Percentage absorption of photon energy from a ${ }^{241}$ Am source. Estimates made using the Monte Carlo method.

\begin{tabular}{|c|c|c|}
\hline \multirow{2}{*}{ Scintillator thickness (mm) } & Absorption (\%) & CsI:Tl* \\
\hline & Mini Crystals & Slice \\
\hline 6 & 100 & 100 \\
\hline 3 & 100 & 100 \\
\hline 2 & 99.6 & 100 \\
\hline 1 & 99.5 & 100 \\
\hline 0.5 & 93.5 & 94.2 \\
\hline 0.25 & 79.9 & 78.6 \\
\hline 0.125 & 59.8 & 60.1 \\
\hline 0.05 & 37.4 & 37.8 \\
\hline 0.025 & 22 & 24.2 \\
\hline 0.01563 & 17.4 & 17.7 \\
\hline 0.008 & 9.6 & 9.7 \\
\hline 0.004 & 5.7 & 5.1 \\
\hline 0.0025 & 3.6 & 3.4 \\
\hline 0.002 & 2.7 & 2.8 \\
\hline 0.001 & 1.3 & 1.5 \\
\hline 0.0005 & 0.6 & 0.7 \\
\hline 0.0001 & 0.1 & 0.1 \\
\hline
\end{tabular}

${ }^{*}$ Absorption of each gamma ray reaching the crystal. 




Figure 7. CsI:Tl mini crystal thickness effect and radiation absorption.



Figure 8. CsI:Tl slice crystal thickness effect and radiation absorption.



Figure 9. Data acquisition system containing one-dimensional photodiode array chips. In the center, a unit of scintillators crystals developed in our laboratory is shown. 
made: (1) CsI:Tl crystal slice and (2) CsI:Tl mini crystals inserted into the spaces of a one-dimensional arrangement, to the photodiode array.

Figure 10 shows, in detail, the coupling of the mini crystals to the chip of the one-dimensional photodiode array.

Electronic board for data acquisition with CCD-type component, using simpler electronic circuitry, Figure 11.

Experiments were conducted with the imager (scanner) using array of photodiodes. Figure 12 and Figure 13 show the results of food casings, where some metallic parts were introduced and the respective result of imaging.

Figure 12 displays the image of a food package (tuna) showing the effect of the insertion of a support containing mini-crystals of CsI:Tl in the imaging system. It is possible to see the image quality, watching the relief of the metal packaging. The X-ray tube was operated at $30 \mathrm{kV}$ to $0.3 \mathrm{~A}$.

Figure 13 shows the image of a food package (bread), presenting the effect of the insertion of a slice of $25 \times 3 \times 2 \mathrm{~mm}$ of the CsI:Tl crystal, in the imaging system. In this experiment, three metal objects with diameters 2,3 and $4 \mathrm{~mm}$ were inserted in the bread package. The X-rays imaging identified these three objects with good resolution. The X-ray tube was operated at $30 \mathrm{kV}$ to $0.3 \mathrm{~A}$.

The result of the imager driven experiment with CCD technology, directly



(a)



(b)

Figure 10. (a) One-dimensional array of photodiodes; (b) Support full with the mini crystals and coupled to the one-dimensional array of photodiodes.



Figure 11. Data acquisition system containing a Sony CCD type Sony chip model ILX551B, 2048 pixel linear CCD sensor (B/W) coupled with the CsI:Tl scintillator crystal developed in our laboratory for this project. 


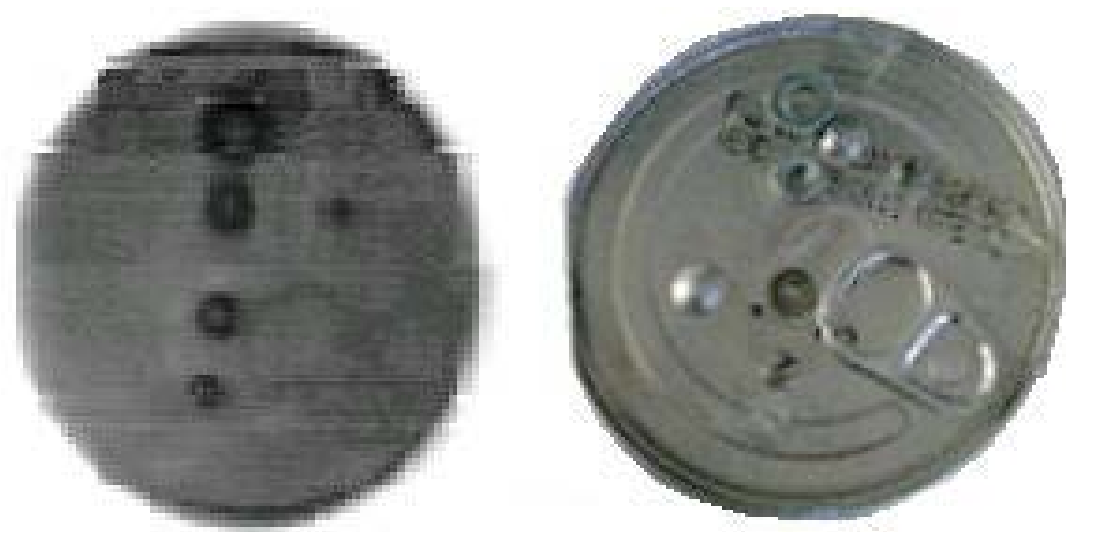

Figure 12. Image of food package showing the effect of the insertion of a support containing mini-crystals of CsI:Tl in the imaging system.


Figure 13. Image of bread package showing the result obtained by inserting a slice of $25 \times$ $3 \times 2 \mathrm{~mm}$ CsI:Tl crystal, in the imaging system. Packing was placed under three iron objects with diameters 2, 3 and $4 \mathrm{~mm}$, seen in the left, highlighted.

coupled to the CsI:Tl scintillator crystal, is shown in Figure 14. In this experiment, a steel washer with dimensions of $12 \times 6 \times 1 \mathrm{~mm}^{3}$ (outside diameter, internal diameter, thickness) has been used.

The good quality of the images obtained in experiments with X-rays imaging using the CsI:Tl mini-crystals and slices of the CsI:Tl crystal, coupled to one-dimensional photodiodes arrangement or coupled photosensor CCD, indicated that this system can be used in quality control in food industry. 


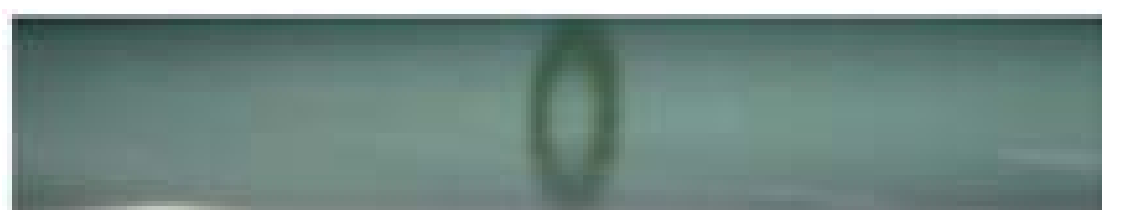

Figure 14. Scanner result in the CCD version of a $12 \times 6 \times 1 \mathrm{~mm}^{3}$ steel washer, using a single CCD unit directly coupled to a strip of $25 \times 3 \times 2 \mathrm{~mm}^{3}$ CsI:Tl crystal.

\section{Conclusions}

The experiments demonstrated that the crystal of cesium iodide doped with thallium grown in our laboratory showed good results, when used in imaging equipment with X-rays.

The result of the analysis of X-ray diffraction on the crystal grown by the vertical Bridgman technique showed a structure compatible with the standard cubic crystalline face centered ( $\mathrm{fcc}$ ).

Slices of the CsI:Tl crystal, with a thickness of $2 \mathrm{~mm}$ and mini crystals in the dimensions of $1 \times 2 \times 3 \mathrm{~mm}^{3}$ may be successfully used in imaging.

By means of estimates obtained by the Monte Carlo method, it has been shown that $2 \mathrm{~mm}$ slices are capable of absorbing $100 \%$ of the energy of the incident X-rays.

Imaging with one-dimensional array of photodiodes and imaging with scanner in CCD version, used with CsI:Tl scintillator crystal, showed efficiency in image quality.

CsI:Tl crystals, although are commercially available, there is the possibility of development of these crystals in our laboratory, which allows obtaining the crystals in different geometric shapes, to meet the need for coupling the photosensor, such as, one-dimensional array of photodiodes and CCD photosensor, with goal of the X-ray imaging.

In continuation of the research will be used the CCD with dimensions $A_{3}(297$ $\mathrm{mm}$ ) directly coupled to a long slice of the crystal scintillator of CsI:Tl covering all its sensitive face, so that it is possible to obtain images of large bodies.

\section{Acknowledgements}

The authors thank FAPESP (Foundation Research of the State of São Paulo) for financial support.

\section{References}

[1] Nikl, M. (2006) Scintillation Detectors for X-Rays. Measurement Science and Technology, 17, 37-54. https://doi.org/10.1088/0957-0233/17/4/R01

[2] Kozyrev, E.A., Kuper, K.E., Lemzyakov, A.G., Petrozhitskiy, A.V. and Popov, A.S. (2016) Performance and Characterization of CsI:Tl Thin Films for X-Ray Imaging Application. Physics Procedia, 84, 245-251.

https://doi.org/10.1016/j.phpro.2016.11.042

[3] Dong, F.Z., Peng, J., Kai, Z.H., Zhen, Z.B., Bo, Q.X., Feng, W.C., Yu, L. and Long, W. (2015) Performance Assessment of CsI(Tl) Screens on Various Substrates for 
X-Ray Imaging. Chinese Physics C, 39, 78202.

https://doi.org/10.1088/1674-1137/39/7/078202

[4] Knoll, G.F. (2010) Radiation Detection and Measurement. 4th Edition, John Wiley \& Sons, New York.

[5] Zhao, W., Ristic, G. and Rowlands, J.A. (2004) X-Ray Imaging Performance of Structured Cesium Iodide Scintillators. Medical Physics, 31, 2594-2605. https://doi.org/10.1118/1.1782676

[6] Cha, B.K., Lee, D.H., Kim, B., Seo, C.W., Jeon, S. and Huh, Y. (2011) High-Resolution X-Ray Imaging Based on Pixel Structured CsI:Tl Scintillating Screens for Indirect X-Ray Image Sensors, Journal of the Korean Physical Society, 59, 3670-3673. https://doi.org/10.3938/jkps.59.3670

[7] Hormozan, Y., Sychugov, L. and Linnros, J. (2016) High Resolution X-Ray Using a Structured Scintillator. Medical Physics, 43, 696-701. https://doi.org/10.1118/1.4939258

[8] Mesquita, C.H. and Hamada, M.M. (2007) Development of Monte Carlo simulation to Study Linear Radiation Position Sensitive Detectors. IEEE Nuclear Science Symposium Conference Record, Honolulu, 26 October-3 November 2007, 871-875. https://doi.org/10.1109/NSSMIC.2007.4436467

[9] Bridgman, P.W. (1925) Certain Physical Properties of Single Crystals of Tungsten, Antimony, Bismuth, Tellurium, Cadmium, Zinc, and Tin. Proceedings of the American Academy of Arts and Sciences, 60, 303-383.

[10] Garapyn, I., Hud, I. and Pavlyk, B. (2004) Properties of Cesium Iodide Prepared by Different Purification Methods. Radiation Measurements, 38, 475-479. https://doi.org/10.1016/j.radmeas.2004.01.014

[11] Margulies, M., Witomski, P. and Duffar, T. (2004) Optimization of the Bridgman Crystal Growth Process. Journal of Crystal Growth, 266, 175-181. https://doi.org/10.1016/j.jcrysgro.2004.02.043

[12] Pereira, M.C.C. and Filho, T.M. (2013) Inorganic Scintillators of $\mathrm{Li}^{+}$Doped CsI for Neutron Detection. International Nuclear Conference (INAC 2013), Recife, 24-29 November 2013.

[13] US Joint Committee on Powder Diffraction Standards. Selected Standard X-Ray Diffraction Powder, Vol. 4, 47. 